\title{
The Sodium Glucose Cotransporter 2 Inhibitor Ipragliflozin Promotes Preferential Loss of Fat Mass in Non-obese Diabetic Goto-Kakizaki Rats
}

\author{
Toshiyuki Takasu,* Yuka Hayashizaki, Jiro Hirosumi, Hideaki Minoura, Nobuaki Amino, \\ Eiji Kurosaki, and Shoji Takakura \\ Tsukuba Research Center, Drug Discovery Research, Astellas Pharma Inc.; 21 Miyukigaoka, Tsukuba, Ibaraki 305- \\ 8585, Japan. \\ Received December 9, 2016; accepted January 27, 2017
}

Sodium glucose cotransporter 2 (SGLT2) inhibitors improve hyperglycemia in patients with type 2 diabetes mellitus (T2DM) by increasing urinary glucose excretion. In addition to their antihyperglycemic effect, SGLT2 inhibitors also reduce body weight and fat mass in obese and overweight patients with T2DM. However, whether or not SGLT2 inhibitors similarly affect body composition of non-obese patients with T2DM remains unclear. In this study, we investigated the effect of the SGLT2 inhibitor ipragliflozin on body composition in a Goto-Kakizaki (GK) rat model of non-obese T2DM. GK rats were treated with ipragliflozin once daily for 9 weeks, starting at 23 weeks of age. Body composition was then analyzed using dual-energy X-ray absorptiometry. Treatment with ipragliflozin increased urinary glucose excretion, reduced hemoglobin A1c (HbA1c) levels and suppressed body weight gain as the dose increased. Body composition analysis revealed that body fat mass was lower in the ipragliflozin-treated groups than in the control group, while lean body mass and bone mineral contents were comparable between groups. Thus, an SGLT2 inhibitor ipragliflozin was found to promote preferential loss of fat mass in a rat model of non-obese T2DM. Ipragliflozin might also promote preferential loss of fat in non-obese patients with T2DM.

Key words ipragliflozin; sodium glucose cotransporter 2 (SGLT2) inhibitor; body composition; anti-diabetic drug; type 2 diabetes mellitus

Type 2 diabetes mellitus (T2DM) is characterized by either or both disturbed insulin secretion from pancreatic $\beta$-cells or insufficient action of insulin (insulin resistance) in peripheral tissues. $^{1,2)}$ Treatment of T2DM typically starts with diet and exercise to reduce excess body weight, which is effective for glycemic control, ${ }^{3)}$ as extra fat is known to increase insulin resistance. ${ }^{4)}$ If glycemic control cannot be effectively achieved through diet and exercise, treatment then progresses to the use of several types of oral antihyperglycemic drug. ${ }^{5,6)}$ However, most of these compounds either induce weight gain (insulin, sulfonylureas, glinides, and thiazolidinediones) or help maintain current weight (metformin, $\alpha$-glucosidase inhibitors, and dipeptidyl peptidase-4 (DPP-4) inhibitors). ${ }^{5,6)}$ Achieving weight loss along with fat loss using such medications has thus far proven difficult. ${ }^{7)}$

Sodium glucose cotransporter 2 (SGLT2) inhibitors have recently been developed as antidiabetic drugs. ${ }^{8,9)}$ SGLT2, a low-affinity and high-capacity glucose transporter, is expressed specifically on the luminal surface of cells in the S1 segment of the proximal tubule ${ }^{10)}$ and reabsorbs approximately $90 \%$ of glucose in the glomerular filtrates in normal glucose-tolerant individuals. ${ }^{8}$ ) SGLT2 inhibitors prevent this reabsorption, thereby increasing urinary glucose excretion and subsequently decreasing blood glucose levels. Treatment with SGLT2 inhibitors improves glycemic parameters and is associated with reduction in body weight in patients with T2DM. ${ }^{11,12)}$ Further, reduction of waist circumference or fat mass has been reported in overweight or obese patients with T2DM. ${ }^{13-15)}$ In animal studies, SGLT2 inhibitors enhanced glucosuria, increased usage of fatty acids instead of glucose as an energy source, and reduced body fat mass in high-fat diet-induced obese (DIO) rats. $^{16-18)}$ However, whether or not SGLT2 inhibitors similarly affect body weight and body composition in non-obese T2DM patients or animal models of T2DM remains unclear.

Goto-Kakizaki (GK) rats, a model of spontaneous nonobese T2DM, were produced from a colony of non-diabetic Wistar rats by selective breeding with glucose intolerance as a selection index. ${ }^{19)}$ The GK rats exhibit decreased $\beta$-cell numbers and function which is accompanied by mild hyperglycemia, impaired glucose-induced insulin secretion, and peripheral insulin resistance. $^{20-24)}$ Here, using dual-energy X-ray absorptiometry (DEXA), we investigated the effect of the selective SGLT2 inhibitor ipragliflozin ${ }^{13,25-29)}$ on body composition in GK rats.

\section{MATERIALS AND METHODS}

Drugs Ipragliflozin L-proline was synthesized at Astellas Pharma Inc. (Ibaraki, Japan). The compound was suspended in $0.5 \%$ methylcellulose solution $(0.5 \% \mathrm{MC})$ as a vehicle and administered by oral gavage at a dosing volume of $5 \mathrm{~mL} / \mathrm{kg}$ of body weight. The dose of ipragliflozin is expressed as the proline-free substance.

Animals and Diets Male GK rats and male Wistar rats aged seven weeks were purchased from CLEA Japan, Inc. (Tokyo, Japan). All animals were housed under specific-pathogen-free conditions in a temperature- and humidity-controlled room with a 12-h light and dark cycle (light on 7:30-19:30). They were fed a standard laboratory chow (CE-2; CLEA Japan, Inc.) and allowed ad libitum access to water. All animal experiments were approved by the Institutional Animal Care 
and Use Committee of Tsukuba Research Center of Astellas Pharma Inc., which is accredited by the Association for Assessment and Accreditation of Laboratory Animal Care International (AAALAC International).

Study Design Body weight of all rats was monitored weekly from age 7 weeks, and the slope of their growth curve showed slowing at around age 20 weeks. At age 23 weeks, the GK rats were divided into 4 groups: diabetic control rats were given vehicle $(n=5)$, and treated rats were given ipragliflozin at $1(n=5), 3(n=4)$, or $10 \mathrm{mg} / \mathrm{kg} / \mathrm{d}(n=4)$. Groups were fashioned so that each had similar mean body weights and hemoglobin A1c (HbAlc) levels. Wistar rats given vehicle served as non-diabetic control animals $(n=5)$. All rats were treated with either ipragliflozin or vehicle once daily in the evening for 9 weeks, starting at 23 weeks of age. Body weight and residual food amount were measured once a week using a digital balance. Eight weeks after initiation of drug administration, blood samples were collected from the tail vein in the morning $(9: 00$ to $10: 00)$ under non-fasting conditions to measure HbA1c, blood glucose, and plasma insulin levels.

Blood Biochemical Assays Blood glucose levels, and plasma insulin levels were measured using Glucose CIITest reagent (Wako Pure Chemical Industries, Ltd., Osaka, Japan) and a rat insulin enzyme-linked immunosorbent assay (ELISA) kit (Shibayagi Co., Ltd., Gunma, Japan), respectively. HbA1c levels were quantified using a DCA2000 System (Siemens, Tokyo, Japan).

Urinary Glucose Excretion At age 31 weeks, spontaneously voided urine samples were collected for $24 \mathrm{~h}$ under self-feeding conditions. Urine volume and urinary glucose concentration were measured, and 24-h urinary glucose excretion was calculated. Urinary glucose concentration was determined using Glucose CII test reagent (Wako Pure Chemical Industries, Ltd.).

Body Composition Body composition was measured at age 32 weeks, after 9 weeks of treatment, with a DEXA system (QDR 2000; Hologic, Waltham, MA, U.S.A.), and results were analyzed using a validated small animal software package (version 7.10; Hologic). ${ }^{30}$ ) This software provides accurate estimations of total body fat mass, total body lean soft tissue mass, total body bone mineral content, and total body mass. Before the measurements, a tissue calibration scan was performed using a Hologic phantom. Each rat was placed on a Plexiglass platform in the prone position under anesthesia with urethane $(1.2 \mathrm{~g} / \mathrm{kg}$, intraperitoneal) for measurement.

Statistical Analysis Results are expressed as mean \pm standard error of mean (S.E.M.). The significance of differences between the non-diabetic control group and diabetic control group were evaluated using Student's $t$-test, while those between the diabetic control group and each ipragliflozin-treated group were analyzed using Dunnett's multiple comparison tests. For each test, statistical significance was defined as a $p$ value less than 0.05 . All data analyses were conducted using SAS statistical software (version 9.1.3; SAS Institute Japan, Ltd., Tokyo, Japan).

\section{RESULTS}

Body Weight and Total Food Intake At age 23 weeks, before starting treatment, mean body weight in the non-diabetic control group was $496.2 \mathrm{~g}$, whereas mean values in the

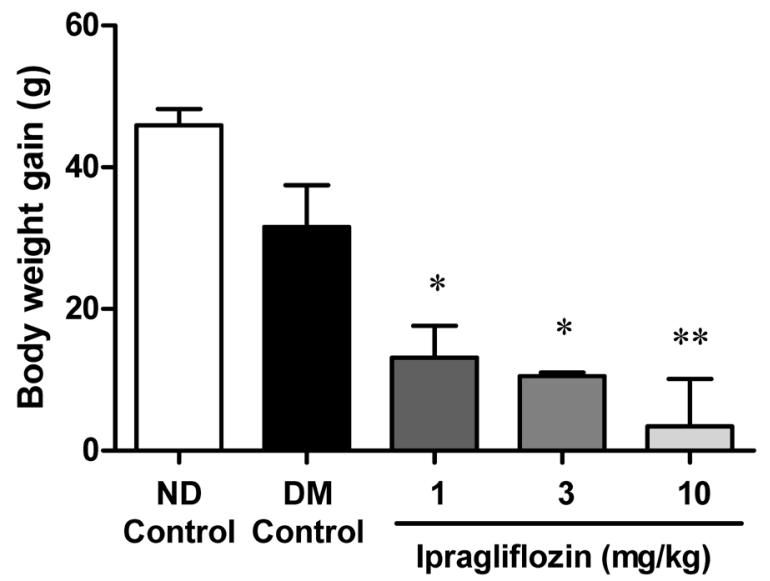

Fig. 1. Effect of Ipragliflozin L-Proline on Body Weight Gain in GK Rats

Body weight gain during the 9 -week treatment period. Values are mean \pm S.E.M of 4 ( 3 and $10 \mathrm{mg} / \mathrm{kg}$ of ipragliflozin-treated group) or 5 (non-diabetic control, diabetic control, and $1 \mathrm{mg} / \mathrm{kg}$ of ipragliflozin-treated group) animals. The significance of differences between the diabetic control group and each ipragliflozintreated group was assessed using Dunnett's multiple comparison test $\left({ }^{*} p<0.05\right.$ and $* * p<0.01)$. Mean body weights of non-diabetic control, diabetic control, and ipragliflozin 1,3, and $10 \mathrm{mg} / \mathrm{kg}$ groups before starting treatment (age 23 weeks) were $496.2,389.7,385.2,384.1$, and $392.2 \mathrm{~g}$, respectively.

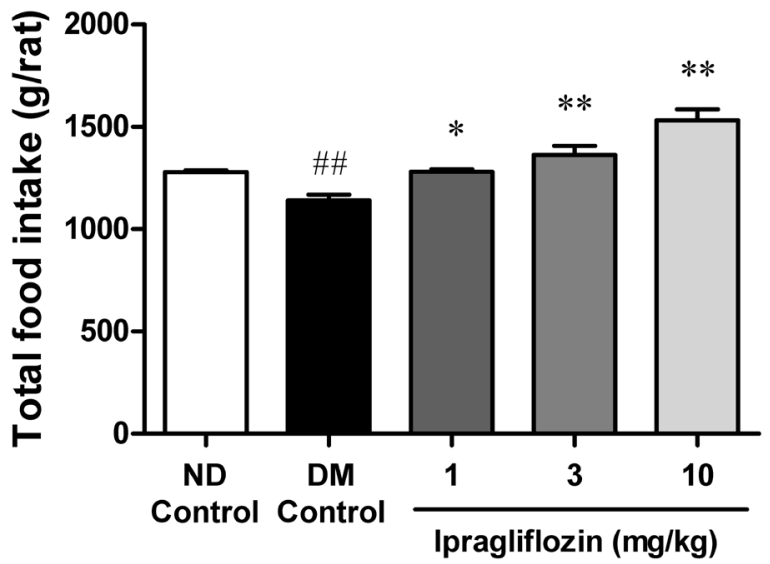

Fig. 2. Effect of Ipragliflozin L-Proline on Total Food Intake in GK Rats

Total food intake during the 9 -week treatment period. Values are mean \pm S.E.M of 4 (3 and $10 \mathrm{mg} / \mathrm{kg}$ of ipragliflozin-treated group) or 5 (non-diabetic control, diabetic control, and $1 \mathrm{mg} / \mathrm{kg}$ of ipragliflozin-treated group) animals. The significance of differences between the non-diabetic and diabetic control groups was determined using Student's $t$-test $(\# \#<0.01)$. The significance of differences between the diabetic control group and each ipragliflozin-treated group was assessed using Dunnett's multiple comparison tests $(* p<0.05$ and $* * p<0.01)$.

diabetic control and ipragliflozin-treated groups ranged from 384.1 to $392.2 \mathrm{~g}$. Mean body weight in the diabetic control group was significantly lower than in the non-diabetic control group. Mean body weights in the non-diabetic and diabetic control groups increased approximately 46 and $32 \mathrm{~g}$, respectively, during the 9-week treatment period. Treatment with ipragliflozin suppressed body weight gain (Fig. 1).

Figure 2 shows total food intake during treatment period. Total food intake in the diabetic control group was significantly lower than in the non-diabetic control group. However, an increase in total food intake was observed in ipragliflozintreated groups. The percentage increases in intake relative to the diabetic control group in the ipragliflozin 1, 3, and $10 \mathrm{mg} / \mathrm{kg} / \mathrm{d}$ groups were $12 \%, 20 \%$, and $35 \%$, respectively.

Blood Biochemical Assays At age 23 weeks, before start- 


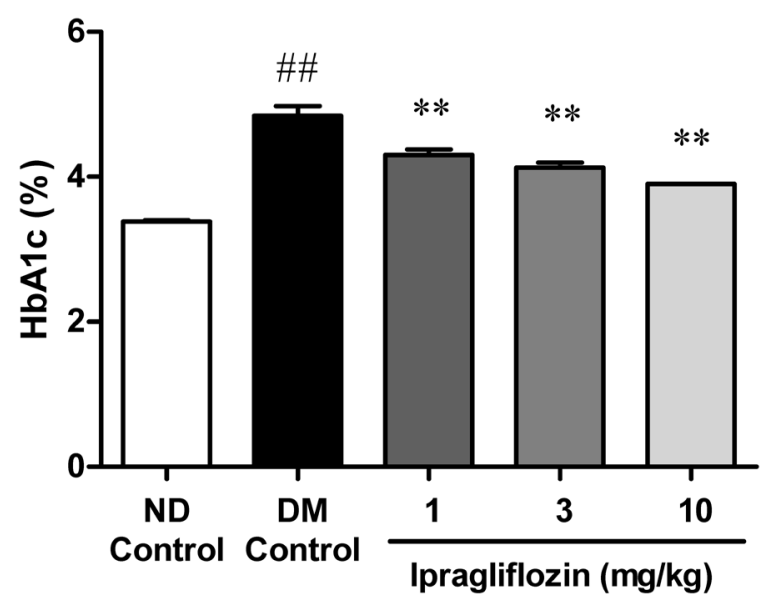

Fig. 3. Effect of Ipragliflozin L-Proline on HbA1c in GK Rats

At age 31 weeks, in Week 8 of treatment, blood samples were collected from the tail vein in the morning $(9: 00$ to $10: 00)$ under non-fasting conditions. Each value represents the mean \pm S.E.M. of 4 ( 3 and $10 \mathrm{mg} / \mathrm{kg}$ of ipragliflozin-treated group) or 5 (non-diabetic control, diabetic control, and $1 \mathrm{mg} / \mathrm{kg}$ of ipragliflozin-treated group) animals. The significance of differences between the non-diabetic and diabetic control groups was determined using Student's $t$-test $\left.{ }^{\# \#} p<0.01\right)$. The significance of differences between the diabetic control group and each ipragliflozin-treated group was assessed using Dunnett's multiple comparison test $(* * p<0.01)$. Mean HbAlc levels of non-diabetic control, diabetic control, and ipragliflozin 1,3 , and $10 \mathrm{mg} / \mathrm{kg}$ groups before starting treatment (age 23 weeks) were $3.38 \%, 4.74 \%, 4.74 \%, 4.78 \%$, and $4.73 \%$, respectively.

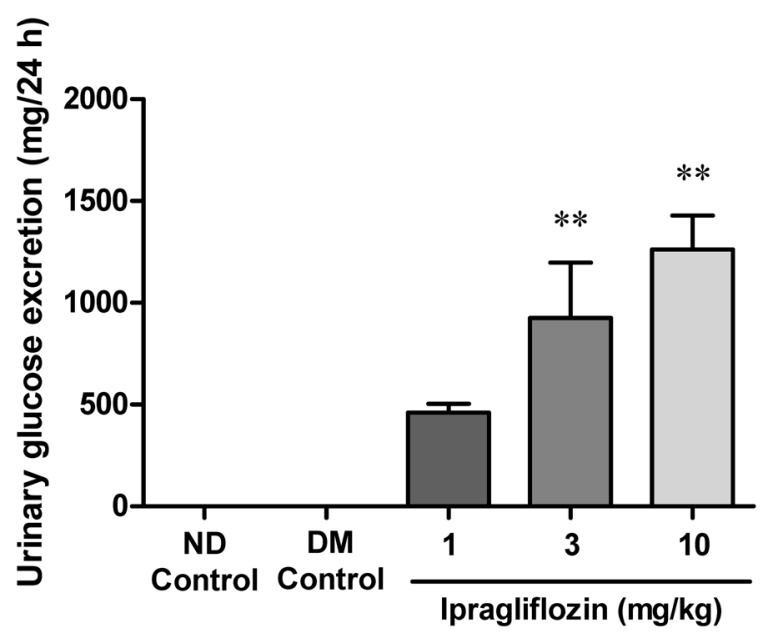

Fig. 4. Effect of Ipragliflozin L-Proline on Urinary Glucose Excretion in GK Rats

Spontaneously voided urine was collected for $24 \mathrm{~h}$ at age 31 weeks, in Week 8 of treatment. Values are mean \pm S.E.M. of $4(3$ and $10 \mathrm{mg} / \mathrm{kg}$ of ipragliflozin-treated group) or 5 (non-diabetic control, diabetic control, and $1 \mathrm{mg} / \mathrm{kg}$ of ipragliflozintreated group) animals. The significance of differences between the diabetic control group and each ipragliflozin-treated group was assessed using Dunnett's multiple comparison test $(* * p<0.01)$

Table 1. Effect of Ipragliflozin on Non-fasting Blood Glucose and Plasma Insulin Levels in GK Rats

\begin{tabular}{ccccccc}
\hline \hline & & & & \multicolumn{3}{c}{ Diabetic } \\
\cline { 4 - 7 } & Age (weeks) & $\begin{array}{c}\text { Non-diabetic Control } \\
(n=5)\end{array}$ & Control $(n=5)$ & $\begin{array}{c}\text { Ipragliflozin } 1 \mathrm{mg} / \mathrm{kg} \\
(n=5)\end{array}$ & $\begin{array}{c}\text { Ipragliflozin } 3 \mathrm{mg} / \mathrm{kg} \\
(n=4)\end{array}$ & $\begin{array}{c}\text { Ipragliflozin } 10 \mathrm{mg} / \mathrm{kg} \\
(n=4)\end{array}$ \\
\hline \multirow{2}{*}{ Glucose $(\mathrm{mg} / \mathrm{dL})$} & 23 & $84.5 \pm 2.9$ & $152.1 \pm 5.8^{\#}$ & $151.4 \pm 6.5$ & $157.8 \pm 23.3$ & $138.6 \pm 1.5$ \\
& 31 & $90.6 \pm 2.5$ & $182.5 \pm 18.5^{\# \#}$ & $156.1 \pm 6.9$ & $182.8 \pm 3.9$ & $165.9 \pm 5.0$ \\
Insulin $(\mathrm{ng} / \mathrm{mL})$ & 23 & $0.97 \pm 0.15$ & $2.08 \pm 0.49$ & $2.79 \pm 0.26$ & $1.90 \pm 0.22$ & $2.17 \pm 0.12$ \\
& 31 & $1.80 \pm 0.51$ & $2.44 \pm 0.48$ & $1.93 \pm 0.18$ & $1.47 \pm 0.23$ & $1.24 \pm 0.09 *$ \\
\hline
\end{tabular}

Ipragliflozin was orally administered to diabetic GK rats once daily for 9 weeks, starting at age 23 weeks. Blood samples were collected at age 23 weeks and 31 weeks. Values are expressed as mean \pm S.E.M. The significance of differences between the non-diabetic and diabetic control groups was determined using Student's $t$-test ( ${ }^{\# \#} p<0.01$ ). The significance of differences between the diabetic control group and each ipragliflozin-treated group was assessed using Dunnett's multiple comparison test $(* p<0.05)$.

ing treatment, mean HbAlc level in the non-diabetic control group was $3.38 \%$, whereas mean values in the diabetic control and ipragliflozin-treated groups ranged from 4.73 to $4.78 \%$. Mean HbAlc levels in the diabetic control group were significantly higher than that of the non-diabetic control group. At age 31 weeks, in Week 8 of treatment, HbA1c levels in the non-diabetic and diabetic control groups were virtually unchanged from levels at age 23 weeks, whereas HbA1c levels in the ipragliflozin-treated groups were lower than those of the diabetic control group (Fig. 3).

Table 1 shows non-fasting blood glucose and plasma insulin levels at age 23 weeks, before starting the treatment, and at age 31 weeks, in Week 8 of treatment. Mean non-fasting blood glucose and plasma insulin levels in the diabetic control group were higher than in the non-diabetic control group. While treatment with ipragliflozin did not affect blood glucose levels at any dose, it did reduce plasma insulin levels as the dose increased.

Urinary Glucose Excretion No urinary glucose excretion was detected in either non-diabetic or diabetic control animals at age 31 weeks. In contrast, treatment with ipragliflozin increased urinary glucose excretion as the dose increased (Fig.

\section{4).}

Body Composition Body composition at age 32 weeks, after the 9-week treatment period, is shown in Table 2. Mean total mass, fat mass, lean mass, and bone mineral content in the diabetic control group were all significantly lower than in the non-diabetic control group. Treatment with ipragliflozin reduced fat mass but did not affect lean mass or bone mineral content.

\section{DISCUSSION}

Here, we investigated the effect of an SGLT2 inhibitor ipragliflozin on body composition of GK rats, a non-obese T2DM model, using the DEXA system, which has high accuracy in measuring total body mass, fat mass, lean mass, and bone mineral content. As expected, treatment of GK rats with ipragliflozin increased urinary glucose excretion and reduced HbAlc levels. Interestingly, body composition analysis revealed that treatment with ipragliflozin decreased fat mass while maintaining both lean mass and bone mineral content. This demonstrates that the SGLT2 inhibitor ipragliflozin promotes preferential loss of fat mass in GK rats. 
Table 2. Effect of Ipragliflozin L-Proline on Total Mass, Fat Mass, Lean Body Mass, and Bone Mineral Content in GK Rats

\begin{tabular}{lccccc}
\hline \hline & \multirow{2}{*}{$\begin{array}{c}\text { Non-diabetic Control } \\
(n=5)\end{array}$} & & \multicolumn{3}{c}{ Diabetic } \\
\cline { 3 - 6 } & & Control $(n=5)$ & $\begin{array}{c}\text { Ipragliflozin } 1 \mathrm{mg} / \mathrm{kg} \\
(n=5)\end{array}$ & $\begin{array}{c}\text { Ipragliflozin } 3 \mathrm{mg} / \mathrm{kg} \\
(n=4)\end{array}$ & $\begin{array}{c}\text { Ipragliflozin } 10 \mathrm{mg} / \mathrm{kg} \\
(n=4)\end{array}$ \\
\hline Total mass $(\mathrm{g})$ & $544.4 \pm 13.7$ & $414.6 \pm 9.2^{\# \#}$ & $389.3 \pm 6.8$ & $383.1 \pm 11.9$ & $387.7 \pm 13.0$ \\
Fat mass $(\mathrm{g})$ & $130.3 \pm 6.5$ & $82.5 \pm 4.6^{\# \#}$ & $67.0 \pm 4.8^{*}$ & $52.3 \pm 3.5^{* *}$ & $51.4 \pm 2.5^{* *}$ \\
Lean mass (g) & $402.7 \pm 8.7$ & $322.9 \pm 7.2^{\# \#}$ & $313.8 \pm 5.5$ & $322.5 \pm 8.9$ & $327.6 \pm 10.5$ \\
Bone mineral content $(\mathrm{g})$ & $11.5 \pm 0.5$ & $9.2 \pm 0.2^{\# \#}$ & $8.6 \pm 0.3$ & $8.3 \pm 0.3$ & $8.7 \pm 0.2$ \\
\hline
\end{tabular}

Ipragliflozin was orally administered to diabetic GK rats once daily for 9 weeks, starting at age 23 weeks. Body composition was measured at age 32 weeks. Values are mean \pm S.E.M. The significance of differences between the non-diabetic and diabetic control groups was determined using Student's $t$-test (\#\# $p<0.01$ ). The significance of differences between the diabetic control group and each ipragliflozin-treated group was assessed using Dunnett's multiple comparison test $(* p<0.05$ and $* * p<0.01)$.

We previously reported that treatment of DIO rats with ipragliflozin caused preferential loss of fat mass. ${ }^{17)}$ In high-fat diet-fed T2DM mice, treatment with ipragliflozin resulted in a decrease in epididymal fat weight as compared to that of vehicle-treated group. ${ }^{31}$ Analysis with indirect calorimetry suggested that the mechanism of loss of fat mass by ipragliflozin in DIO rats was via enhanced lipolysis and fatty acid utilization. ${ }^{17)}$ Other SGLT2 inhibitors, namely dapagliflozin, tofogliflozin, canagliflozin and empagliflozin, have been reported to reduce the fat mass ${ }^{16,18,32-34)}$ which was associated with decreased respiratory quotient values in animal studies, ${ }^{16,18,33)}$ suggesting that SGLT2 inhibitors promote fatty acid utilization. These previous findings therefore suggest that the preferential loss of fat mass in the present study was due to the promotion of fatty acid utilization over glucose as an energy source, triggered by calorie loss associated with enhanced glucosuria. Further, the preferential loss of fat mass might be mainly attributable to suppressed body weight gain, which was observed in ipragliflozin-treated groups. In spite of the suppression of body weight gain, total food intake in the ipragliflozin-treated groups was higher than in the diabetic control group. This increase in intake may be a compensatory response against calorie loss through enhanced urinary glucose excretion induced by ipragliflozin, since similar findings have been reported using DIO rats, where the SGLT2 inhibitors dapagliflozin and tofogliflozin induced persistent urinary glucose excretion accompanied by adaptive or compensatory hyperphagia. ${ }^{16,18)}$

Accumulation of fat, especially visceral fat, is of particular concern as a causal factor of insulin resistance in not only obese but also non-obese patients with T2DM. ${ }^{35-37)}$ As such, reduction of body fat mass would aid in treating T2DM. In a clinical study of overweight patients with T2DM, treatment with ipragliflozin induced modest weight loss accompanied by a reduction in fat mass, while also improving glycemic control. ${ }^{14,15)}$ Analysis of body composition revealed that the reduction of body fat was the largest contributor to the reduction in body weight. ${ }^{15)}$ Dapagliflozin also reduced total body weight in obese patients with T2DM, predominantly by reducing total-body fat mass, wherein the decrease in visceral fat mass was greater than that in subcutaneous fat mass. ${ }^{38,39)}$ Merovci et al. further demonstrated that two weeks' treatment with dapagliflozin improved muscle insulin sensitivity in obese patients with T2DM. ${ }^{40}$ However, no study has yet investigated the benefits of SGLT2 inhibitors on fat accumulation and insulin resistance in non-obese patients with T2DM. In the present study, treatment with ipragliflozin decreased plasma insulin levels in addition to the reduction in fat mass, which might indicate that ipragliflozin improves insulin resistance in GK rats. Additional studies are needed to confirm the insulin resistance-improving effect of ipragliflozin. Further, future studies should clarify the clinical effectiveness of SGLT2 inhibitors on insulin resistance in non-obese patients with T2DM.

In the present study, in which blood samples were collected in the morning, treatment with ipragliflozin elicited significant reduction in HbAlc levels but did not reduce non-fasted blood glucose levels in GK rats. Ipragliflozin was given just before the dark period to suppress elevation of blood glucose levels during feeding, as food intake in GK rats is larger during the dark period than that during the light period (data not shown). This suppression of blood glucose elevation during the feeding period is assumed to have resulted in the reduced HbAlc levels observed in GK rats treated with ipragliflozin.

In conclusion, in the GK rat model of non-obese T2DM, the SGLT2 inhibitor ipragliflozin improved hyperglycemia while simultaneously reducing fat mass with no reduction in lean body mass or bone mineral content. These results suggest that SGLT2 inhibitor ipragliflozin might similarly promote preferential loss of fat mass in non-obese patients with T2DM.

Acknowledgments This study was conducted at Tsukuba Research Center, Drug Discovery Research, Astellas Pharma Inc. The authors thank Drs. Atsuo Tahara and Masanori Yokono for their valuable comments and encouragement.

Conflict of Interest The authors are employees of Astellas Pharma Inc.

\section{REFERENCES}

1) Abdul-Ghani MA, Tripathy D, DeFronzo RA. Contributions of beta-cell dysfunction and insulin resistance to the pathogenesis of impaired glucose tolerance and impaired fasting glucose. Diabetes Care, 29, 1130-1139 (2006).

2) DeFronzo RA. Insulin resistance, lipotoxicity, type 2 diabetes and atherosclerosis: the missing links. The Claude Bernard Lecture 2009. Diabetologia, 53, 1270-1287 (2010).

3) Palermo A, Maggi D, Maurizi AR, Pozzilli P, Buzzetti R. Prevention of type 2 diabetes mellitus: is it feasible? Diabetes Metab. Res. Rev., 30 (Suppl. 1), 4-12 (2014).

4) Sattar N, Gill JM. Type 2 diabetes as a disease of ectopic fat? BMC Med., 12, 123 (2014).

5) Eckel RH, Kahn SE, Ferrannini E, Goldfine AB, Nathan DM, Schwartz MW, Smith RJ, Smith SR, Obesity and type 2 diabetes: what can be unified and what needs to be individualized? Diabetes Care, 34, 1424-1430 (2011).

6) Aguilar RB. Evaluating treatment algorithms for the management of 
patients with type 2 diabetes mellitus: a perspective on the definition of treatment success. Clin. Ther., 33, 408-424 (2011).

7) Pi-Sunyer FX. Weight loss in type 2 diabetic patients. Diabetes Care, 28, 1526-1527 (2005).

8) Abdul-Ghani MA, Norton L, Defronzo RA. Role of sodium-glucose cotransporter 2 (SGLT 2) inhibitors in the treatment of type 2 diabetes. Endocr. Rev., 32, 515-531 (2011).

9) Wilding JP. The role of the kidneys in glucose homeostasis in type 2 diabetes: clinical implications and therapeutic significance through sodium glucose co-transporter 2 inhibitors. Metabolism, 63, 1228-1237 (2014).

10) Chen J, Williams S, Ho S, Loraine H, Hagan D, Whaley JM, Feder JN. Quantitative PCR tissue expression profiling of the human SGLT2 gene and related family members. Diabetes Ther., 1, 57-92 (2010).

11) Vallon V. The mechanisms and therapeutic potential of SGLT2 inhibitors in diabetes mellitus. Annu. Rev. Med., 66, 255-270 (2015).

12) Rajeev SP, Cuthbertson DJ, Wilding JP. Energy balance and metabolic changes with sodium-glucose co-transporter 2 inhibition. Diabetes Obes. Metab., 18, 125-134 (2016).

13) Kashiwagi A, Kazuta K, Yoshida S, Nagase I. Randomized, placebo-controlled, double-blind glycemic control trial of novel sodium-dependent glucose cotransporter 2 inhibitor ipragliflozin in Japanese patients with type 2 diabetes mellitus. J. Diabetes Investig., 5, 382-391 (2014).

14) Kashiwagi A, Kazuta K, Goto K, Yoshida S, Ueyama E, Utsuno A. Ipragliflozin in combination with metformin for the treatment of Japanese patients with type 2 diabetes: ILLUMINATE, a randomized, double-blind, placebo-controlled study. Diabetes Obes. Metab., 17, 304-308 (2015).

15) Iizuka $T$, Iemitsu K, Takihata M, Takai M, Nakajima S, Minami N, Umezawa S, Kanamori A, Takeda H, Kawata T, Ito S, Kikuchi T, Amemiya H, Kaneshiro M, Mokubo A, Takuma T, Machimura H, Tanaka K, Asakura T, Kubota A, Aoyagi S, Hoshino K, Ishikawa M, Matsuzawa Y, Obana M, Sasai N, Kaneshige H, Minagawa F, Saito T, Shinoda K, Miyakawa M, Tanaka Y, Terauchi Y, Matsuba I. Efficacy and safety of ipragliflozin in Japanese patients with type 2 diabetes: Interim outcome of the ASSIGN-K study. J. Clin. Med. Res., 8, 116-125 (2016).

16) Devenny JJ, Godonis HE, Harvey SJ, Rooney S, Cullen MJ, Pelleymounter MA. Weight loss induced by chronic dapagliflozin treatment is attenuated by compensatory hyperphagia in diet-induced obese (DIO) rats. Obesity, 20, 1645-1652 (2012).

17) Yokono M, Takasu T, Hayashizaki Y, Mitsuoka K, Kihara R, Muramatsu Y, Miyoshi S, Tahara A, Kurosaki E, Li Q, Tomiyama H, Sasamata M, Shibasaki M, Uchiyama Y. SGLT2 selective inhibitor ipragliflozin reduces body fat mass by increasing fatty acid oxidation in high-fat diet-induced obese rats. Eur. J. Pharmacol., 727, 66-74 (2014)

18) Suzuki M, Takeda M, Kito A, Fukazawa M, Yata T, Yamamoto M, Nagata T, Fukuzawa T, Yamane M, Honda K, Suzuki Y, Kawabe Y. Tofogliflozin, a sodium/glucose cotransporter 2 inhibitor, attenuates body weight gain and fat accumulation in diabetic and obese animal models. Nutrition \& Diabetes, 4, e125 (2014).

19) Goto Y, Kakizaki M, Masaki N. Production of spontaneous diabetic rats by repetition of selective breeding. Tohoku J. Exp. Med., 119, 85-90 (1976).

20) Bisbis S, Bailbe D, Tormo MA, Picarel-Blanchot F, Derouet M, Simon J, Portha B. Insulin resistance in the GK rat: decreased receptor number but normal kinase activity in liver. Am. J. Physiol., 265, E807-E813 (1993).

21) O'Rourke CM, Davis JA, Saltiel AR, Cornicelli JA. Metabolic effects of troglitazone in the Goto-Kakizaki rat, a non-obese and normolipidemic rodent model of non-insulin-dependent diabetes mellitus. Metabolism, 46, 192-198 (1997)

22) Ueta K, Ishihara T, Matsumoto $Y$, Oku A, Nawano M, Fujita T,
Saito A, Arakawa K. Long-term treatment with the $\mathrm{Na}+$-glucose cotransporter inhibitor T-1095 causes sustained improvement in hyperglycemia and prevents diabetic neuropathy in Goto-Kakizaki Rats. Life Sci., 76, 2655-2668 (2005).

23) Movassat J, Bailbe D, Lubrano-Berthelier C, Picarel-Blanchot F, Bertin E, Mourot J, Portha B. Follow-up of GK rats during prediabetes highlights increased insulin action and fat deposition despite low insulin secretion. Am. J. Physiol. Endocrinol. Metab., 294, E168-E175 (2008).

24) Portha B, Lacraz G, Kergoat M, Homo-Delarche F, Giroix MH, Bailbe D, Gangnerau MN, Dolz M, Tourrel-Cuzin C, Movassat J. The GK rat beta-cell: a prototype for the diseased human beta-cell in type 2 diabetes? Mol. Cell. Endocrinol., 297, 73-85 (2009).

25) Tahara A, Kurosaki E, Yokono M, Yamajuku D, Kihara R, Hayashizaki Y, Takasu T, Imamura M, Qun L, Tomiyama H, Kobayashi Y, Noda A, Sasamata M, Shibasaki M. Pharmacological profile of ipragliflozin (ASP1941), a novel selective SGLT2 inhibitor, in vitro and in vivo. Naunyn Schmiedeberg's Arch. Pharmacol., 385, 423-436 (2012)

26) Takasu T, Hayashizaki Y, Tahara A, Kurosaki E, Takakura S. Antihyperglycemic effect of ipragliflozin, a sodium-glucose cotransporter 2 inhibitor, in combination with oral antidiabetic drugs in mice. Clin. Exp. Pharmacol. Physiol., 42, 87-93 (2015).

27) Veltkamp SA, van Dijk J, Collins C, van Bruijnsvoort M, Kadokura T, Smulders RA. Combination treatment with ipragliflozin and metformin: a randomized, double-blind, placebo-controlled study in patients with type 2 diabetes mellitus. Clin. Ther., 34, 1761-1771 (2012).

28) Fonseca VA, Ferrannini E, Wilding JP, Wilpshaar W, Dhanjal P, Ball G, Klasen S. Active- and placebo-controlled dose-finding study to assess the efficacy, safety, and tolerability of multiple doses of ipragliflozin in patients with type 2 diabetes mellitus. J. Diabetes Complications, 27, 268-273 (2013).

29) Wilding JP, Ferrannini E, Fonseca VA, Wilpshaar W, Dhanjal P, Houzer A. Efficacy and safety of ipragliflozin in patients with type 2 diabetes inadequately controlled on metformin: a dose-finding study. Diabetes Obes. Metab., 15, 403-409 (2013).

30) Engelbregt MJ, van Weissenbruch MM, Popp-Snijders C, Lips P, Delemarre-van de Waal HA. Body mass index, body composition, and leptin at onset of puberty in male and female rats after intrauterine growth retardation and after early postnatal food restriction. Pediatr. Res., 50, 474-478 (2001).

31) Tahara A, Kurosaki E, Yokono M, Yamajuku D, Kihara R, Hayashizaki Y, Takasu T, Imamura M, Li Q, Tomiyama H, Kobayashi Y, Noda A, Sasamata M, Shibasaki M. Effects of SGLT2 selective inhibitor ipragliflozin on hyperglycemia, hyperlipidemia, hepatic steatosis, oxidative stress, inflammation, and obesity in type 2 diabetic mice. Eur. J. Pharmacol., 715, 246-255 (2013).

32) Millar PJ, Pathak V, Moffett RC, Pathak NM, Bjourson AJ, O'Kane MJ, Flatt PR, Gault VA. Beneficial metabolic actions of a stable GIP agonist following pre-treatment with a SGLT2 inhibitor in high fat fed diabetic mice. Mol. Cell. Endocrinol., 420, 37-45 (2016).

33) Liang Y, Arakawa K, Ueta K, Matsushita Y, Kuriyama C, Martin T, Du F, Liu Y, Xu J, Conway B, Conway J, Polidori D, Ways K, Demarest K. Effect of canagliflozin on renal threshold for glucose, glycemia, and body weight in normal and diabetic animal models. PLOS ONE, 7, e30555 (2012).

34) Vickers SP, Cheetham SC, Headland KR, Dickinson K, Grempler R, Mayoux E, Mark M, Klein T. Combination of the sodiumglucose cotransporter-2 inhibitor empagliflozin with orlistat or sibutramine further improves the body-weight reduction and glucose homeostasis of obese rats fed a cafeteria diet. Diabetes Metab. Syndr. Obes. Target Ther., 7, 265-275 (2014).

35) Pouliot MC, Despres JP, Nadeau A, Moorjani S, Prud'Homme D, Lupien PJ, Tremblay A, Bouchard C. Visceral obesity in men. Associations with glucose tolerance, plasma insulin, and lipoprotein 
levels. Diabetes, 41, 826-834 (1992).

36) Bu J, Feng Q, Ran J, Li Q, Mei G, Zhang Y. Visceral fat mass is always, but adipokines (adiponectin and resistin) are diversely associated with insulin resistance in Chinese type 2 diabetic and normoglycemic subjects. Diabetes Res. Clin. Pract., 96, 163-169 (2012).

37) Taniguchi A, Nakai Y, Sakai M, Yoshii S, Hamanaka D, Hatae Y, Kawata M, Yamanouchi K, Okumura T, Doi K, Tokuyama K, Nagasaka S, Fukushima M. Relationship of regional adiposity to insulin resistance and serum triglyceride levels in nonobese Japanese type 2 diabetic patients. Metabolism, 51, 544-548 (2002).

38) Bolinder J, Ljunggren O, Kullberg J, Johansson L, Wilding J, Langkilde AM, Sugg J, Parikh S. Effects of dapagliflozin on body weight, total fat mass, and regional adipose tissue distribution in patients with type 2 diabetes mellitus with inadequate glycemic control on metformin. J. Clin. Endocrinol. Metab., 97, 1020-1031 (2012)

39) Bolinder J, Ljunggren O, Johansson L, Wilding J, Langkilde AM, Sjostrom CD, Sugg J, Parikh S. Dapagliflozin maintains glycaemic control while reducing weight and body fat mass over 2 years in patients with type 2 diabetes mellitus inadequately controlled on metformin. Diabetes Obes. Metab., 16, 159-169 (2014).

40) Merovci A, Solis-Herrera C, Daniele G, Eldor R, Fiorentino TV, Tripathy D, Xiong J, Perez Z, Norton L, Abdul-Ghani MA, DeFronzo RA. Dapagliflozin improves muscle insulin sensitivity but enhances endogenous glucose production. J. Clin. Invest., 124, 509-514 (2014). 